\title{
SUB-MILLIARCSECOND OPTICAL ASTROMETRY AND BINARY STARS
}

\author{
J.T. ARMSTRONG \\ Universities Space Research Association \\ NRL/USNO Optical Interferometer Project \\ U. S. Naval Observatory - AD 5 \\ 3450 Massachusetts Ave. NW, Washington, DC 20392, USA \\ tarmstr@atlas.usno.navy.mil
}

\begin{abstract}
Long-baseline optical interferometry has made it possible to measure the visual orbits of binary stars with major axes as small as 5 mas and errors of $\lesssim 100 \mu$ as. Interferometers now nearing completion will extend these values to $a \gtrsim 500 \mu$ as and $\sigma_{a} \sim 10 \mu$ as. Observations of double-lined spectroscopic binaries with current interferometers have already yielded some mass estimates with precisions rivaling those from fitting the light curves of eclipsing double-lined systems. Luminosity estimates based on combined visual interferometric observations and velocity curves are often more precise than those from more indirect methods based on estimates of $T_{\text {eff }}$. New interferometers now coming into operation will make it possible to measure fundamental parameters in dozens to hundreds of binary systems.
\end{abstract}

\section{Introduction}

The high angular resolution of the new optical interferometers coming into operation at several sites around the world will considerably expand our knowledge of binary stars. One area that will benefit is the determination of fundamental stellar quantities-masses, radii, luminosities, and metallicities. Stellar masses can be measured only in binaries, and until recently only in eclipsing, double-lined spectroscopic binaries (SB2s) could we measure masses and radii to the $1 \%$ to $2 \%$ precision needed for comparison with stellar evolution models (Andersen 1991). 
The results from the Mark III interferometer on Mt. Wilson, California, discussed in these proceedings by C. Hummel, demonstrate that masses and radii can be measured in non-eclipsing SB2s, and show that the distance, and thus the luminosities, can be measured as well. A few of these results already meet the demanding standards set by the results from eclipsing systems. The new interferometers will further expand the systems available for these measurements to include fainter SB2s, and to include nearby SB1s.

\section{Measurement of Fundamental Stellar Parameters}

Until recently, measurements of fundamental stellar parameters that are precise enough to challenge models of stellar evolution have been limited to detached, eclipsing, double-lined spectroscopic binaries (Andersen 1991). These were the only systems in which it has been possible to determine the mass ratio and the semimajor axis $a$ (in $\mathrm{km}$ ) of the orbit from the velocity amplitudes $K_{1}$ and $K_{2}$, and to determine the orbital inclination $i$ from modeling the shape of the light curve when the stars eclipse one another. With the orbital eccentricity $e$ also deduced from the velocity curves, and with the period $P$ in hand, the masses $\mathcal{M}_{1,2}$ of the components are then calculated. An added benefit of eclipsing systems is that the lightcurve modeling also produces estimates of the radii $R_{1,2}$ (also in $\mathrm{km}$ ) of the components. However, the distance $D$ to the system is not measured; rather, it is derived from the estimated effective temperatures $T_{\text {eff }}$, the radii, and the apparent magnitude $m_{V}$. Eclipsing systems, then, yield direct determinations of stellar masses and radii, and an indirect determination of the luminosity $L$.

As detailed by Andersen (1991), 45 such systems have given us results that are sufficiently precise to challenge stellar evolution models. His criteria of precision are masses and radii with $<2 \%$ mean errors, although he accepts $3.5 \%$ errors for YY Gem, the only well-studied M system. But these systems occupy only part of the HR diagram, being concentrated on the main sequence; only five components have $\mathcal{M}<1 \mathcal{M}_{\odot}$, only eight are of spectral type later than $\mathrm{F}$, and only two are red giants. Late-type dwarfs suffer from two selection effects, as pointed out by Popper (1993) among others: because they are small, they must be close together in order to be likely to eclipse; and because they are faint, they are less often discovered and harder to observe. Among binaries with giant components, the magnitude difference $\Delta m$ is often too large for spectral lines of both to be measured adequately, unless they are sufficiently close in mass that they become giants at about the same time. 


\section{Interferometric Results}

There are many more non-eclipsing binaries than eclipsing binaries, and it has always been possible to combine visual and spectroscopic orbits to obtain masses in non-eclipsing systems. But the overlap between the classes of visual and spectroscopic binaries has until recently been small. Using visual orbits, rather than eclipse light curves, to determine the orbital inclinations affords, among other things, a measurement of the distance to the system: the spectroscopic data give the physical size of the orbit to within a factor of $\sin i$, while the visual data give the angular scale and $i$. The disadvantage of visual data in comparison with eclipse light curves is that the stellar radii are not determined.

Speckle interferometry, and recently separate-element interferometry, have enlarged the overlap between these two classes (e.g., Griffin 1992). Several groups built two-element interferometers during the 1970s and 1980s, with typical baselines of a few tens of meters and apertures less than $10 \mathrm{~cm}$. Of these, the Mark III interferometer on Mt. Wilson was the most productive, in terms of amount of data acquired. It was also unique in having a dual purpose: astrometry over the whole (available) sky, and "imaging," i.e., measurement of stellar diameters and binary orbits. The single-star astrometry of the Mark III was not precise enough to measure astrometric orbits of close binaries, but binary data from the Mark III has made contributions to the list of stars with precisely known fundamental quantities.

The Mark III, which operated on Mt. Wilson from 1986 to 1992, was a two-element Michelson interferometer with active fringe and angle tracking, automated operations, and 450 to $800 \mathrm{~nm}$ wavelength coverage. The $5 \mathrm{~cm}$ maximum aperture limited observations to $m_{V} \lesssim 5^{\mathrm{m}}$. Astrometric observations used one of two fixed $12 \mathrm{~m}$ baselines and provided stellar positions to 20 mas precision (Hummel et al. 1994b) over a $90^{\circ} \times 30^{\circ}(\alpha \times \delta)$ region. The variable baselines of 3 to $31.5 \mathrm{~m}$ made it possible to measure stellar diameters larger than 2.5 mas with 0.05 to 0.1 mas precision (Mozurkewich et al. 1991, 1994), and binary orbits larger than 3 mas with 0.05 mas precision. The Mark III used a single $250 \mathrm{~nm}$ wide channel near $\lambda 700 \mathrm{~nm}$ for fringe phase tracking, and typically three channels, each $25 \mathrm{~nm}$ wide, at $\lambda \lambda 500$, 550 , and $800 \mathrm{~nm}$, for scientific data. The calibration precision of the fringe visibilities ranged from $1 \%$ to a few percent in the $800 \mathrm{~nm}$ channel, to as much as $15 \%$ in the $450 \mathrm{~nm}$ channel; for the binary observations, this made it possible to measure orbits of systems with $\Delta m \lesssim 33^{\mathrm{m}} 5$, and to measure $\Delta m$ in those systems to a precision of 0.05 to 0.3 .

The Mark III obtained more than two dozen visual orbits with milliarcsecond or sub-milliarcsecond precision. Seventeen of these are double-lined spectroscopic binaries; of these, results have been published for five ( $\beta$ Ari, 
$\alpha$ Equ, $\phi$ Cyg, $\eta$ And, $\alpha$ Aur, $\beta$ Per) and are in preparation for six more (Hummel et al. 1994c). It is worth remarking on some of these systems in the context of what is possible from interferometric observations. In a few of them, the precision of the masses is of the order of a few percent, or some components are in a part of the HR diagram where masses have not been well measured.

\subsection{CAPELLA}

The Mark III data (Hummel et al. 1994a) confirm and refine the visual orbit of the well-known giant system Capella (G1 III + G8-K0 III), one of the best-observed visual systems; they also demonstrate the accuracy of optical interferometry. The masses obtained agree well with those of Barlow et al. (1993) and have mean errors of $2.2 \%$ and $1.6 \%$ for components $\mathrm{Aa}$ and $\mathrm{Ab}$. The $\Delta m$ values from the Mark III are also consistent with other results. The limb-darkened diameters $\theta_{\text {ld }}=8.5 \pm 0.1$ mas $(1.2 \%)$ and $6.4 \pm 0.3$ mas (4.4\%), with the size of Aa reported for the first time. From the size of the orbit measured with the Mark III, the orbital parallax $\pi_{\text {orb }}=0$ ". $0751 \pm 0$ ".0005 $(0.7 \%)$, giving $\log \left(L / L_{\odot}\right)=1.895 \pm 0.007$ and $1.890 \pm 0.015$. (The smallest value of $\sigma_{\log L}$ in Andersen 1991 is 0.02.) If the mass ratio (which has been problematic in the past) has finally been well determined, Capella can be added to the list of stars with precisely known masses, radii, and luminosities.

\section{2. $\phi$ CYGNI}

This giant system (K0 III + K0 III) yielded $3 \%$ mass estimates in Mark III observations (Armstrong et al. 1992b); this is a slightly lower precision than Andersen (1991) recommends, but few masses of giants are known. The estimated angular diameters are 1 mas or less, so no diameter measurement with the Mark III was possible. For $\phi$ Cygni, $\sigma_{\mathcal{M}_{1}}$ and $\sigma_{\mathcal{M}_{2}}$ are dominated by $\sigma_{K_{1}}$ and $\sigma_{K_{2}}$, while the uncertainties in the absolute magnitude $M_{V}$ ( 0.11 and 0.13 for the more- and less-massive components, corresponding to 0.044 and 0.052 in $\sigma_{\log L}$ ) is dominated by $\sigma_{\Delta m}$; reducing this uncertainty depends on improved calibration of the fringe contrast, since it is difficult to measure $\Delta m$ precisely when it is near zero. The diameters were not measured; Armstrong et al. (1992a) estimated uniform-disk diameters of 0.94 and 0.82 mas in the $V$ band.

In order for $\phi$ Cygni to satisfy Andersen's criteria for precisely measured stars, the masses should be improved and the angular diameters measured. In this case, $\sigma_{\mathcal{M}}$ is dominated by $\sigma_{K_{1,2}}$, which are taken by Armstrong et al. (1992a) to be four times the uncertainties given by Rach \& Herbig (1961) due to a discrepancy between the visual and spectroscopic values 
of $e$; thus, this system could benefit by another spectroscopic study. For measurements of the stellar diameters, there is no solution except to build a bigger interferometer.

\section{3. $\beta$ AURIGAE}

$\beta$ Aurigae (Hummel et al. 1994c) is an eclipsing system that already appears in Andersen's list, with mean errors in $R_{1,2}$ of $2.1 \%$, in $\mathcal{M}_{1,2}$ of $1.1 \%$, and in $L_{1,2}$ of $19 \%$. The Mark III data improve the distance determination to $24.9 \pm 0.5 \mathrm{pc}(2 \%)$, but $\sigma_{L}$ is dominated by $\sigma_{\Delta m}$, which remains about 0.2 . As with $\phi$ Cygni, improving $\Delta m$ will require improved calibration.

\section{4. $\alpha$ EQUULEI}

$\alpha$ Equulei (Armstrong et al. 1992a) (G0 III + A5 V) fails to appear on the list of systems with precisely known parameters for two reasons: $\sigma_{\sin i} / \sin i=3.5 \%$, and $\sigma_{K_{A}} / K_{A}=4 \%$. With the improved data reduction introduced by Hummel et al. (1993), Hummel and Armstrong (1993) give $\sigma_{\sin i} / \sin i=1.6 \%$, alleviating the situation somewhat. However, the velocities of the A star in this system are difficult to measure, since the $G$ spectrum must be subtracted before the radial velocities can be measured (Stickland 1976; Rosvick \& Scarfe 1991).

\section{The New Interferometers}

These examples demonstrate a variety of observational challenges that must be resolved in order to obtain improved values of fundamental stellar parameters, including from resolving the disks of the components (Capella, $\phi$ Cygni, $\alpha$ Equulei), which requires longer baselines; improving the measurements of the visual orbit ( $\alpha$ Equulei), which requires longer baselines and/or better calibration; improving the determination of $\Delta m$ ( $\beta$ Aurigae), which also requires better calibration; and improving radial velocity measurements ( $\phi$ Cygni, $\alpha$ Equulei). Except for the velocity measurements, all these challenges can be met by improved and enlarged interferometers, such as those now coming into operation.

As Table 1 shows, the typical interferometer of the 1990s has considerably larger apertures and/or longer baselines, and more collecting apertures, than the interferometers of the 1970s and 1980s. These improvements should lead to more than an order of magnitude improvement in angular resolution and to observations of $8^{\mathrm{m}}$ or $9^{\mathrm{m}}$ stars. My discussion will focus on the NPOI interferometer, which has the largest number of elements and the largest two-dimensional layout, and which is the one with which I am most familiar. 
TABLE 1. New interferometers after 1990

\begin{tabular}{|c|c|c|c|c|c|}
\hline Year & Interferometer & Apertures & $\begin{array}{l}\text { Max. } \\
\text { BL }\end{array}$ & $\lambda(\mu \mathrm{m})$ & Ref. \\
\hline 1990 & $\begin{array}{l}\text { COAST (Cam- } \\
\text { bridge University) }\end{array}$ & $4 \times 40 \mathrm{~cm}$ & 100 & $0.4-0.95$ & Cox (1993) \\
\hline 1992 & $\begin{array}{l}\text { SUSI } \\
\text { (Sydney University) }\end{array}$ & $2 \times 14 \mathrm{~cm}$ & 640 & $0.4-0.85$ & Davis (1993) \\
\hline 1994 & $\begin{array}{l}\text { IOTA (Center } \\
\text { for Astrophysics) }\end{array}$ & $3 \times 45 \mathrm{~cm}$ & 38 & $\begin{array}{l}0.45-0.8 \\
2.2\end{array}$ & $\begin{array}{l}\text { Carleton } \\
\text { et al. (1994) }\end{array}$ \\
\hline 1994 & $\begin{array}{l}\text { NPOI astrometric } \\
\text { (USNO/NRL) }\end{array}$ & $\begin{array}{l}4 \times 12.5 \mathrm{~cm} \\
(35 \mathrm{~cm} \text { in } 1995)\end{array}$ & 38 & $0.45-0.9$ & Hutter (1994) \\
\hline 1994 & $\begin{array}{l}\text { NPOI imaging } \\
\text { (USNO/NRL) }\end{array}$ & $\begin{array}{l}6 \times 12.5 \mathrm{~cm} \\
(35 \mathrm{~cm} \text { planned })\end{array}$ & 437 & $0.45-0.9$ & $\begin{array}{l}\text { Armstrong } \\
\text { (1994) }\end{array}$ \\
\hline $\begin{array}{l}1995 \\
\text { planned }\end{array}$ & $\begin{array}{l}\text { ASEPS-0 } \\
\text { (JPL) }\end{array}$ & $2 \times 40 \mathrm{~cm}$ & 100 & 2.2 & $\begin{array}{l}\text { Colavita } \\
\text { et al. (1994) }\end{array}$ \\
\hline
\end{tabular}

The NPOI consists of co-located astrometric and imaging arrays that share the feed system, optics laboratory, control system, and the designs for most of the components. The feed system supports a $12 \mathrm{~cm}$ beam; beam-compressing telescopes with $35 \mathrm{~cm}$ apertures are currently planned for installation in the astrometric array in 1995 or 1996, and in the imaging array some time after that.

The astrometric array, described in these proceedings by Hutter, Johnston, \& Mozurkewich, has four siderostats as the array elements, one at the center and three at the ends of $20 \mathrm{~m}$ arms, for a maximum baseline of $38 \mathrm{~m}$. The siderostats are permanently mounted, and their motions at the micron level due to bearing errors and to diurnal and seasonal temperature variations will be monitored by a laser metrology truss. The initial goal is a catalog of about 1000 stars with positional errors of 2 mas, on a par with the expected precision of the HIPPARCOS catalog.

The imaging array will consist of six siderostats that can be moved among a set of 30 stations placed along the arms of a $\mathrm{Y}$ with $250 \mathrm{~m}$ arms, with either two or three siderostats per arm in any given configuration. The standard configurations will have siderostats equally spaced along the arms. Tracking fringes on the short baselines between neighboring siderostats will make it possible to track fringes on the longest baselines even when the fringe contrast on those baselines is too low to detect in real time. The initial observing programs will include synthesis of images of the surfaces of nearby red giants, and, as with the Mark III, stellar diameter and binary 
orbit measurements.

Both arrays will detect fringes by feeding the spectrally dispersed light $(\lambda \lambda 450$ to $850 \mathrm{~nm})$ from each baseline into a $1 \times 32$ array of fibers, each leading to an avalanche photo-diode. This technique will give the NPOI two additional advantages over the Mark III. One is that fringe contrast calibration will be improved by the availability of data all across the visible band, making it possible to measure $\Delta m$ better and detect both components in systems with larger $\Delta m$ than was possible with the Mark III. The other advantage is that using multiple narrow bands, rather than a single wide band, will make it possible to track the fringe packet, not just the fringe phase modulo $2 \pi$ as with the Mark III, making fringe detection and tracking more robust.

\section{Prospects of New Interferometric Observations}

With these improvements in interferometry in mind, we can estimate the observational capabilities of the imaging array and of the other new interferometers by extrapolation from the Mark III. With a maximum baseline of $437 \mathrm{~m}$, the NPOI imaging array should be able to measure orbits as small as $250 \mu$ as with errors as small as $7 \mu$ as, and diameters as small as $180 \mu$ as with errors of 4 to $7 \mu$ as. The NPOI should perform somewhat better than this extrapolation because of the improved calibration and fringe tracking, and because up to 15 baselines will be used simultaneously; but I will ignore those sources of improvement. To be conservative, I will take the limits to be $a>400 \pm 10 \mu$ as and $\theta>200 \pm 10 \mu$ as. Even so, these extended capabilities will open up a large range of binary systems in which fundamental stellar parameters can be precisely determined.

\subsection{ECLIPSING, DOUBLE-LINED DETACHED SPECTROSCOPIC BINARIES}

Nineteen of the 45 systems listed by Andersen (1991) are both bright enough $\left(m<9^{\mathrm{m}}\right)$ to observe and have orbits $(a>400 \mu$ as $)$ that can be resolved by the NPOI; for all of these, $\sigma_{a}<10 \mu$ as will improve the distance determinations. In systems with shallow eclipses for which the determinations of $i$ and stellar radii from the light-curve solution have correlated errors, the measurement of the inclination from the visual orbit may improve the radius determinations as well. The new interferometers are also capable of directly measuring the angular diameters of 14 stars in ten of the Andersen (1991) systems; for both components of $\beta$ Aurigae and YY Geminorum, $10 \mu$ as precision will improve the diameter determinations over the values listed in Andersen (1991), providing a further check on the light-curve models. 
These measurements would have two effects. First, the determinations of the luminosities of the stars, from distances determined from the angular and physical orbit sizes, would be direct and more precise. Second, they would make possible a refinement of the calibration of the $T_{\text {eff }}$ scale, from angular sizes determined directly from the interferometry.

\subsection{NON-ECLIPSING DOUBLE-LINED BINARIES}

McAlister (1985) counted the systems in the 7th Catalogue of Spectroscopic Binaries that could be reached with an interferometer with a $300 \mathrm{~m}$ baseline. Using the estimated separations at nodal passage of Halbwachs (1981), he counted 180 systems north of $\delta=0^{\circ}$, with 102 wider than 1 mas. I have done the same exercise with the 8th Catalogue (Batten, Fletcher, \& MacCarthy 1989). I calculated expected angular separations from the apparent magnitudes, and absolute magnitudes roughly estimated from the spectral types and luminosity classes. This procedure is accurate to no better than a factor of two, but for our purposes, that is sufficient. Selecting for SB2s with , I find 201 systems. Of these, 130 systems have separations less than 3 mas, and 42 systems are fainter than $5^{\mathrm{m}}$; these 172 are thus entirely new to interferometric observation. An approximate HR diagram of the 201 systems is shown in Figure 1, in which the absolute magnitudes have been roughly estimated from the spectral types.

\subsection{NON-ECLIPSING SINGLE-LINED BINARIES}

If the astrometric precision is great enough, another category of spectroscopic binary becomes accessible to precise determination of stellar parameters. For SB2s, the physical scale is given by $K_{1,2}$ and $P$. For SB1s, we may substitute $\pi_{\text {trig }}$ and $a$ (in arc seconds) for the missing $K_{2}$. We will need $0.5 \%$ precision in $\pi_{\text {trig }}$ in order to get $2 \%$ precision in $\mathcal{M}_{1}$ and $1 \%$ precision in $\mathcal{M}_{2}$. If the NPOI's astrometric precision with repeated observations and/or over small angles on the sky improves by an order of magnitude from our estimate for initial operations of 2 mas (which only experience will show), we can observe SB1s out to $25 \mathrm{pc}$ with the possibility of obtaining precise results. The Gliese catalog of stars within 25 pc (Gliese \& Jahreiss 1993) contains $130 \mathrm{SBs}$ with $\delta>-10^{\circ}$ and $m<9^{\mathrm{m}}$, while the Batten catalog contains $32 \mathrm{SB} 1$ s with $\delta>-10^{\circ}$ whose primaries are G dwarves brighter than $7 \mathrm{~m} 5$ or $\mathrm{K}$ dwarves brighter than 8.5 , and thus are within roughly $25 \mathrm{pc}$ as well. 


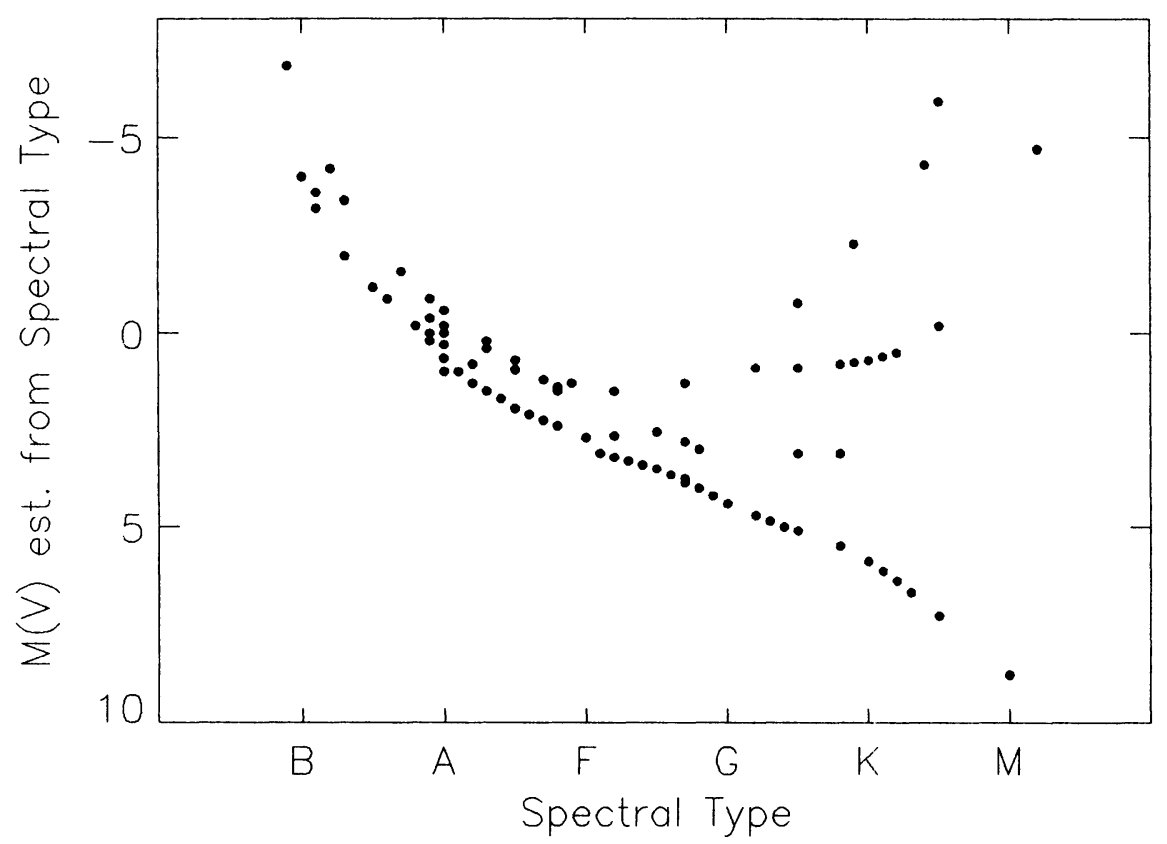

Figure 1. HR diagram of SB2s from the catalog of Batten, Fletcher, \& MacCarthy (1989) resolvable with the NPOI imaging array. Selection criteria were $m<9^{\mathrm{m}}$, orbital qualities a, b, or c, and angular separations $\left(a_{1}+a_{2}\right) \sin i>0.4$ mas. $M_{V}$ were roughly estimated from spectral types.

\section{Conclusion}

I have touched on only one line of research the precise determination of stellar parameters to which high-precision astrometry in binary systems can make a contribution. Interferometry with separate apertures has started to live up to its promise as a tool for precise astrometry, having completed the list of precisely known parameters (in the sense of Andersen 1991) for one system (i.e., $R_{1,2}$ in Capella). Many more scientific contributions from interferometry are to be expected from the interferometers now coming into operation, with their longer baselines, increased collecting area, and multiple baselines.

\section{Acknowledgements}

This work was done in collaboration with D.F. Buscher, C.S. Denison, N.M. Elias II, C.A. Hummel, D.J. Hutter, K.J. Johnston, D. Mozurkewich, A. Quirrenbach, and R.S. Simon. 


\section{References}

Andersen, J. 1991, A\&ARev, 3, 91

Armstrong J.T. 1994, in Amplitude and Intensity Spatial Interferometry II, SPIE Proc. 2200, ed. J.B. Brekinridge, p. 62

Armstrong, J.T., Hummel, C.A., Quirrenbach, A., Buscher, D.F., Mozurkewich, D., Vivekanand, M., Simon, R.S., Denison, C.S., Johnston, K.J., Pan X.-P., Shao, M., \& Colavita, M.M. 1992a, AJ, 104, 2217

Armstrong, J.T., Mozurkewich, D., Vivekanand, M., Simon, R.S., Denison, C.S., Johnston, K.J., Pan X.-P., Shao, M., \& Colavita, M.M. 1992a, AJ, 104, 241

Barlow, D.J., Fekel, F.C., \& Scarfe, C.D. 1993, PASP, 105, 476

Batten, A.H., Fletcher, J.M., \& MacCarthy, D.G. 1989, Publ. DAO, 17, 1

Carleton, N.P., Traub, W.A., Lacasse, M.G., Nisenson, P., Pearlman, M.R., Reasenberg, R.D., Xu, X., Coldwell, C.M., Panasyuk, A., Benson, J.A., Papaliolios, C., Predmore, R., Schloerb, F.P., Dyck, H.M., \& Gibson, D.M. 1994, in Amplitude and Intensity Spatial Interferometry II, SPIE Proc. 2200, ed. J.B. Brekinridge, p. 152

Colavita, M.M., Shao, M., Hines, B.E., Wallace, J.K., Gursel, Y., Malbet, F., Yu, J.W., Singh, H., Beichman, C.A., Pan X.-P., Nakajima, T., \& Kulkarni, S.R. 1994, in Amplitude and Intensity Spatial Interferometry II, SPIE Proc. 2200, ed. J.B. Brekinridge, p. 89

Cox, G.C. 1993, in Very High Angular Resolution Imaging, IAU Symp. 158, ed. J.G. Robinson and W.J. Tango, p. 163

Davis, J. 1993, in Very High Angular Resolution Imaging, IAU Symp. 158, ed. J.G. Robinson and W.J. Tango, p. 163

Gliese, W., \& Jahreiss, H. 1993, Third Catalogue of Nearby Stars (preliminary version), available on the Astronomical Data Center CD ROM

Griffin, R.F. 1992, in Complementary Approaches to Double and Multiple Star Research, IAU Colloquium 135, ed. H.A. McAlister and W.I. Hartkopf (ASP Conf. Ser., San Francisco), p. 98

Halbwachs, J.L. 1981, A\&ASupp, 44, 47

Hummel, C.A., Armstrong, J.T., Quirrenbach, A., Buscher, D.F., Mozurkewich, D., Simon, R.S., \& Johnston, K.J. 1993, AJ, 106, 2486

Hummel, C.A., Armstrong, J.T., Quirrenbach, A., Buscher, D.F., Mozurkewich, D., Elias, N.M. II, \& Wilson, R.E. 1994a, AJ, 107, 1859

Hummel, C.A., Mozurkewich, D., Elias, N.M. II, Quirrenbach, A., Buscher, D.F., Armstrong, J.T., Johnston, K.J., Simon, R.S., \& Hutter, D.J. 1994b, AJ, 108, 326

Hummel, C.A., et al. 1994c, in preparation

Hutter, D.J. 1994, in Amplitude and Intensity Spatial Interferometry II, SPIE Proc. 2200, ed. J.B. Brekinridge, p. 71

McAlister, H.A. 1985, in Calibration of Fundamental Stellar Quantities, IAU Symp. 111, ed. D.S. Hayes, L.E. Pasinetti, and A.G. Davis Phillip (Reidel, Dordrecht), p. 97

Mozurkewich, D., et al. 1994, in preparation

Mozurkewich, D., Johnston, K.J., Simon, R.S., Bowers, P.F., Gaume, R.A., Hutter, D.J., Colavita, M.M., Shao, M., \& Pan X.-P. 1991, AJ, 101, 2207

Popper, D.M. 1993, ApJLett, 404, L67

Rach, R.A., \& Herbig, G.H. 1961, ApJ, 133, 143

Rosvick, J.M., \& Scarfe, C.D. 1991, MNRAS, 252, 68

Stickland, D.J. 1976, MNRAS, 175, 473 